\title{
Elastic-plastic effect study in hypervelocity impact by SPH method
}

\author{
Xiangfei Gong ${ }^{1,2, a}$, Shudao Zhang $^{2}$, and Jiming Yang ${ }^{1}$ \\ ${ }^{1}$ University of Science and Technology of China, 230022 Hefei, China \\ ${ }^{2}$ Institute of Applied Physics and Computational Mathematics, 100094 Beijing, China
}

\begin{abstract}
A new distributed parallel SPH programming scheme using AMR (adaptive mesh refinement) background grid is proposed, in which the size of a grid is decided based on maximal smoothed length of local particles. In detonation simulation, computational complexity of the new scheme is better than $\mathrm{O}(\mathrm{NlgN})$ which is the best order of magnitude in the previous methods. Several hypervelocity impact problems are simulated using the new SPH scheme with state equations or strength model. The simulation results are discussed comparison to experimental and computed results in other literature.
\end{abstract}

\section{Introduction}

Numerical simulation is increasingly becoming a significant means for engineering application and science research. In spacecraft protection problems the velocity of impact objects is generally higher than ten kilometers per second. In the computer simulation of this kind of hypervelocity impact issue Lagrangian methods seem to be more favored than Euler methods because of the development of meshfree Lagrangian algorithms which aren't puzzled by large deformations [1].

For centuries, numerical methods based on grid or mesh play the essential roll in computer simulation, despite the great success, numerical method based on grid suffer from severe difficulties in some aspects including free surface, deformable boundary, moving interface, large deformation, etc. Over the past years meshfree methods, e.g. smoothed particle hydrodynamics (SPH), have been an allimportant research focus of more effective numerical methods for complicated problems [2]. In SPH method, the materials in computational domain are represented by discrete particles, which possess of material properties and have generally unalterable mass. The particles possessing different material properties naturally trace the moving interface along with the movement of the particle. There are no fixed links between particles in SPH that allows a straightforward handling of large deformations, on the other hand that makes it necessary to find new neighboring particles at every step, which is very time-consuming so that SPH is commonly highly inefficient compared with grid based methods. The inefficiency is a crucial matter that restricts extensive application of SPH, so it's very important to improve the neighbor particles search efficiency in SPH method [3].

The algorithm in this paper references to tree structure of link-list method [4] and variable size of bucket method [5]. Background grid of adaptive mesh refinement (AMR) [6] is implemented as buckets that the new search algorithm can be regarded as a modified bucket search

${ }^{a}$ Corresponding author: gong_xiangfei@iapcm.ac.cn algorithm with tree-structure buckets. Size of background grids of the new algorithm is variable that it can be applied when smoothing length is alterable, while higher search efficiency can be gained by the ordering between buckets when finding neighbor buckets.

In the paper hypervelocity impact examples are calculated using the new SPH algorithm with materials described by state equations or by strength model. And the simulation results are discussed comparison to experimental data.

\section{SPH algorithm with AMR background grid}

In this paper, SPH method is used for the numerical simulation and the extensive presentation can be found in [7]. Here some noticeable contents of SPH method are addressed.

\subsection{Governing equations in $\mathrm{SPH}$}

After kernel and particle approximation, for Euler equations controlling the ideal fluid dynamic problems, we have

$$
\begin{gathered}
\frac{d \rho_{i}}{d t}=\sum_{j=1}^{N} m_{j} v_{i j}^{\beta} \cdot \frac{\partial W_{i j}}{\partial x_{i}^{\beta}} \\
\frac{d v_{i}^{\alpha}}{d t}=-\sum_{j=1}^{N} m_{j}\left(\frac{p_{i}}{\rho_{i}^{2}}+\frac{p_{j}}{\rho_{j}^{2}}\right) \frac{\partial W_{i j}}{\partial x_{i}^{\alpha}} \\
\frac{d e_{i}}{d t}=\frac{1}{2} \sum_{j=1}^{N} m_{j}\left(\frac{p_{i}}{\rho_{i}^{2}}+\frac{p_{j}}{\rho_{j}^{2}}\right) v_{i j}^{\beta} \frac{\partial W_{i j}}{\partial x_{i}^{\beta}} .
\end{gathered}
$$

Where $\rho$ denotes the scale density, $m$ is mass of a particle, $v$ is velocity, $W$ is kernel function, $p$ is static pressure and $e$ is internal energy pre unit mass.

This is an Open Access article distributed under the terms of the Creative Commons Attribution License 4.0, which permits unrestricted use, distribution, and reproduction in any medium, provided the original work is properly cited. 
For Navier-Stokes(N-S) equations controlling the general fluid dynamics problems, we have

$$
\begin{gathered}
\frac{d \rho_{i}}{d t}=\sum_{j=1}^{N} m_{j} v_{i j}^{\beta} \cdot \frac{\partial W_{i j}}{\partial x_{i}^{\beta}} \\
\frac{d v_{i}^{\alpha}}{d t}=-\sum_{j=1}^{N} m_{j}\left(\frac{\sigma_{i}^{\alpha \beta}}{\rho_{i}^{2}}+\frac{\sigma_{j}^{\alpha \beta}}{\rho_{j}^{2}}\right) \frac{\partial W_{i j}}{\partial x_{i}^{\alpha}} \\
\frac{d e_{i}}{d t}=\frac{1}{2} \sum_{j=1}^{N} m_{j}\left(\frac{p_{i}}{\rho_{i}^{2}}+\frac{p_{j}}{\rho_{j}^{2}}\right) v_{i j}^{\beta} \frac{\partial W_{i j}}{\partial x_{i}^{\beta}}+\frac{\mu_{i}}{2 \rho_{i}} \varepsilon_{i}^{\alpha \beta} \varepsilon_{j}^{\alpha \beta} .
\end{gathered}
$$

Where $\sigma$ denotes the total stress tensor and $\varepsilon$ denotes the strain tensor.

\subsection{Equations of state (EOS) and constitution model}

In computational simulation, the projectile and target is provided by Mie-Gruneisen equation of state. Elasticity is implemented incrementally and the Johnson-Cook strength model [8] describes von Mises plasticity as a function of strain, strain rate and temperature,

$$
\sigma=\left[A+B \varepsilon^{n}\right]\left[1+C \ln \dot{\varepsilon}^{*}\right]\left[1-T^{* m}\right] .
$$

Where $\sigma$ is the von Mises tensile flow stress, $\varepsilon$ is the equivalent plastic strain, $\dot{\varepsilon}^{*}=\dot{\varepsilon} / \dot{\varepsilon}_{0}^{*}$ is the dimensionless plastic strain rate for $\dot{\varepsilon}_{0}=1.0 \mathrm{~s}^{-1}$, and $T^{*}$ is the homologous temperature.

\section{3. $\mathrm{SPH}$ smoothing function}

Cubic B-spline smoothing function is adopted in simulation which is maybe the most frequently used smoothing function:

$$
W(R, h)=\alpha_{d} \times \begin{cases}\frac{2}{3}-R^{2}+\frac{1}{2} R^{3}, & 0 \leq R<1, \\ \frac{1}{6}(2-R)^{3}, & 1 \leq R<2, \\ 0, & R \geq 2 .\end{cases}
$$

In one-, two- and three-dimensional space, $\alpha_{d}=1 / h$, $15 / 7 \pi h^{2}$ and $3 / 2 \pi h^{3}$, respectively. Gauss kernel and quintic B-spline is also tried, while the results are almost the same.

\subsection{AMR background grid}

\subsubsection{Mesh form}

In background grid search algorithm, the uniform size of background grid is restricted by the maximal smoothing length of particles $l>\max \left(\kappa h_{i}\right) \quad i=1, \ldots, N$, where $N$ is the number of all particles.

In AMR background grids, size of the jth grid can be restricted as $\max \left(\kappa h_{i}\right)<l_{j}<2 \alpha \max \left(\kappa h_{i}\right) \quad i=1, N_{j}$, $\alpha>1.0$ and $N_{j}$ is the number of particles in the jth grid.

What should be paid attention to is that grids whose coarser father grid are just the same in $2^{\mathrm{d}}$ tree mesh structure can be combined to one grid. In the AMR grid configurations, neighbor particles of a given particle can be found in its situated grid or in grids of the same size adjoining to the aforementioned grid.

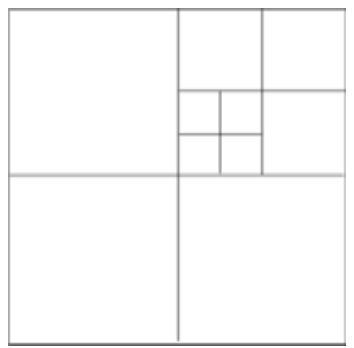

Figure 1. Background grid of adaptive mesh refinement.

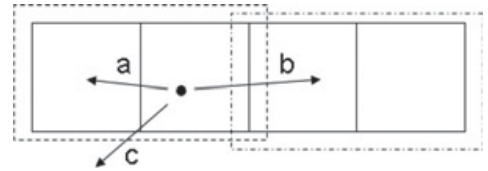

Figure 2. Particle movement.

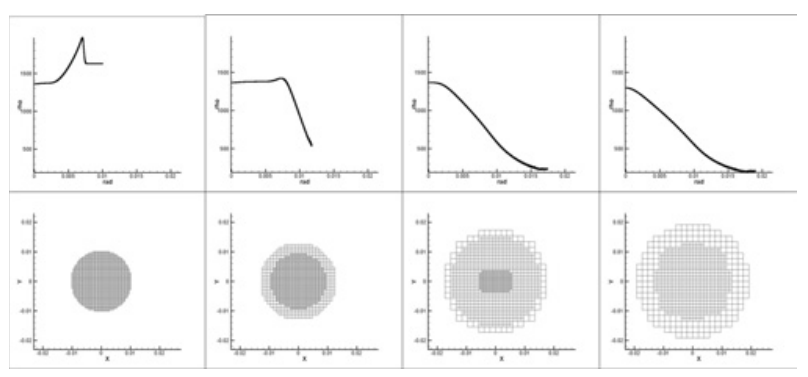

Figure 3. Density and background grids at different computational step.

\subsubsection{Particle movement and creating new grid}

A particle must belong to a new grid when its new location isn't in the primary situated grid. There are three cases, (a) the new grid is already exist, and it is situated in the same course as the primary grid, (b) the new grid is situated in a different course, (c) the new grid is maybe not exist then it should be created. Correspondingly, (a) the particle is transferred to the link-list of the new grid, (b) the particle is transferred to the new course, (c) a new grid is created in the course where the primary grid is situated.

\section{Simulation results}

\subsection{D explosive detonation}

Two-dimensional explosive detonation is simulated by AMR background grid SPH. TNT explosive is presented by JWL equations of state, detonation velocity $D_{C J}=$ $6930 \mathrm{~m} /$ sand detonation pressure $P_{C J}=2.1 \times 10^{10} \mathrm{~Pa}$.

Figure 3 shows the density distributing and grids at different computational moment, which demonstrates the background grid evolvement.

Evolvement of $\mathrm{C} / \mathrm{N}$ is showed in Fig. 4, in which $\mathrm{C}$ denotes the searching amount and $\mathrm{N}$ denotes the particle amount, so the $\mathrm{C} / \mathrm{N}$ denotes the average number of searching needed by each particle. It shows that the evolvement rule is the same in spite of different particle amount.

The comparison of $5 \mathrm{~N} / \mathrm{M}$ and $\mathrm{C} / \mathrm{N}$ is showed in Fig. 5, where $\mathrm{M}$ denotes the background grid amount and 


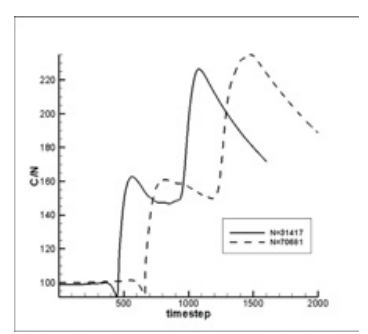

Figure 4. Evolvement of $\mathrm{C} / \mathrm{N}$.

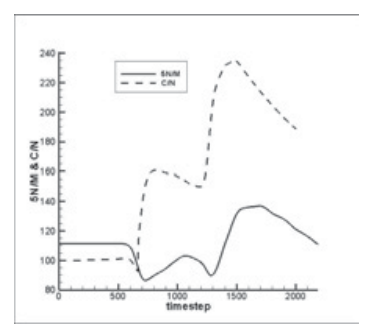

Figure 5. Comparison of $5 \mathrm{~N} / \mathrm{M}$ and $\mathrm{C} / \mathrm{N}$.

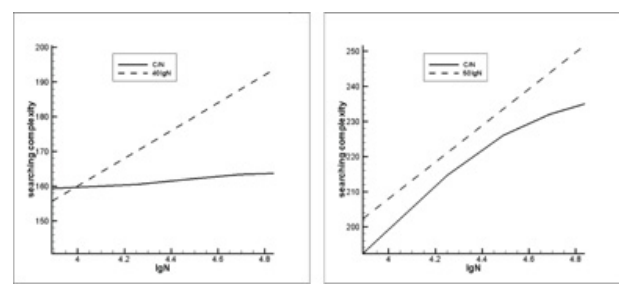

Figure 6. Vertex of $\mathrm{C} / \mathrm{N}$ and $\lg (\mathrm{N})$ in level -1 and level -2 .

$\mathrm{N} / \mathrm{M}$ denotes the average number of particles in each grid. The same trends show the influence of N/M to $\mathrm{C} / \mathrm{N}$ that $\mathrm{C} / \mathrm{N}$ increases and reduces along with the N/M.

In Fig. 6 the dashed line, $\mathrm{mlg}(\mathrm{N})$, increases faster than the real line, $\mathrm{C} / \mathrm{N}$, while $\mathrm{N}$ increases. Here $\mathrm{m}$ is a constant. Then we get $\mathrm{C} / \mathrm{N}<\mathrm{mlg}(\mathrm{N})$, i.e. $\mathrm{C}<\mathrm{mNlg}(\mathrm{N})$, in the order of magnitude, $\mathrm{C}<\mathrm{O}(\mathrm{N} l g \mathrm{~N})$.

\subsection{Sphere impacting a single plate}

The aforementioned computational approach is used to simulate the impact of a sphere onto a plate at $6.15 \mathrm{~km} / \mathrm{s}$. The diameter of the sphere is $15.88 \mathrm{~mm}$, and the thickness of the plate is $0.772 \mathrm{~mm}$. The sphere material is 2017-T4 aluminum and the plate material is 6061-T6 in experimentation, they are both substituted as 2024-T351 aluminum in computational simulation because the two model parameters are not available while are similar in composition and response as 2024-T351 aluminum.

In Fig. 7 the colored images show the computed results at $2.5,4.0$ and $8.1 \mu \mathrm{s}$ after impact. Computed results with just state equations are almost the same as that with constitution model. The achromatic images are from experimental radiograph at $0.0,8.1$ and $23.2 \mu \mathrm{s}$ in literature [1]. The computed sphere shape are similar to the experimental shape but are obviously flattened in the impact direction and there are more neat corners which show the insufficiency of strength in local area. There are two possible causes of this disagreement, the constitution model is not enough or the SPH method is too smoothed to keep local rigidity.

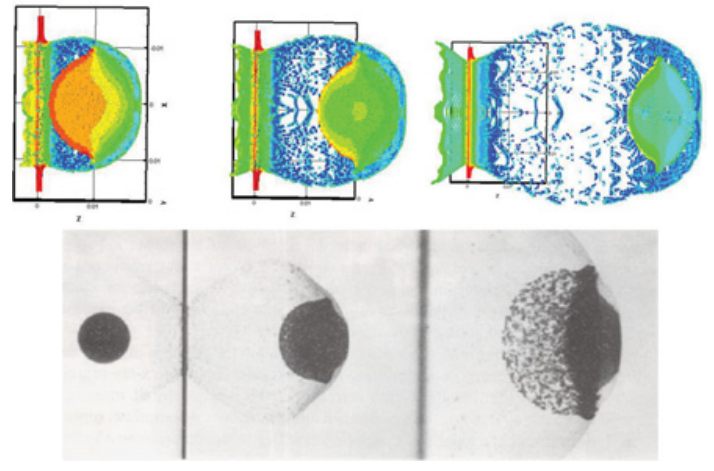

Figure 7. A sphere impact a single plate.

I
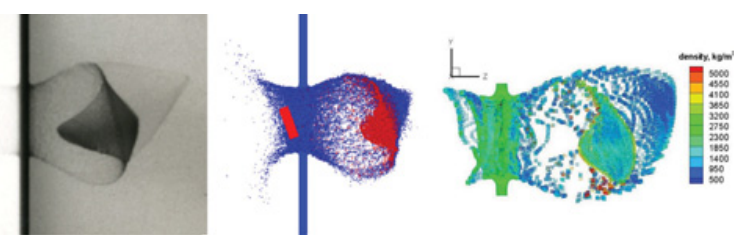

Figure 8. Oblique cylinder impact a single plate.

\subsection{Oblique cylinder impacting a single plate}

An OFHC copper cylinder impacts onto a 6061-T6 aluminum plate. The impact velocity is $6.47 \mathrm{~km} / \mathrm{s}$, and the obliquity is $20.6^{\circ}$. The cylinder diameter is $7.72 \mathrm{~mm}$, and its length is $2.36 \mathrm{~mm}$. The plate thickness is $2.03 \mathrm{~mm}$.

In Fig. 8 the left is radiograph, the middle is computed result in [1] and the right is computed result in the aforementioned computational approach. In the result of [1] projectile material is colored red, and target material blue, while projectile and target material are displayed according to density grading in results of this paper. The computed debris-cloud results are similar to the computed results in [1]. The size of backward cone is underpredicted in the same way while the forward cone is more close to the test data. Computed results with state equations are almost the same as results with constitution model in the same way.

\section{Conclusions}

A new SPH algorithm with AMR background grid is proposed referencing to link-list and bucket method, and the neighbor particle searching efficiency is improved. By comparison to the experimental data, the computational approach is showed to be feasible to hypervelocity impact simulations. From the computed results the following conclusions can be gotten that impact materials can be described well enough by state equations in hypervelocity impact problems and that the constitution model in this paper is maybe not enough or the SPH method is not suitable for local details description of elastic-plastic effect in hypervelocity impact.

\section{References}

[1] S.R. Beissel, C.A. Gerlach, G.R. Johnson. Int. J. Impact Eng., 33 (2006)

[2] M.B. Liu, G.R. Liu, Arch. Comput. Method E., 17 (2010) 
[3] W.S. Yang, X.Y. Meng, Z.H. Wang, Ship Science and Technology, 34, 12 (2012)

[4] J.J. Monaghan, J.C. Lattanzio, Astron. Astr., 149 (1985)

[5] G.R. Liu, Z.H. Tu, Comput. Method Appl. M., 191 (2002)
[6] X.F. Gong, S.D. Zhang, S. Jiang, Comput. Phys., 4 (2006) (in Chinese)

[7] G.R. Liu, M.B. Liu, Smoothed particle hydrodynamics: a meshfree method. (Hunan University, Changsha, 2005)

[8] G.R. Johnson, W.H. Cook, Eng. Fract. Mech., 21, 1 (1985) 